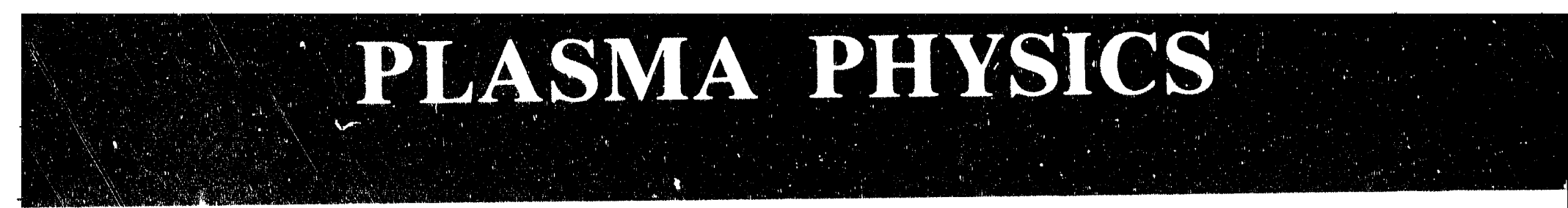

LOCKED MODES AND MAGNETIC FIELD ERRORS IN MST

A.F. ALMAGRI, S. ASSADI, S.C. PRAGER, J.S. SARFF, D.W. KERST

DOE/ER/53198-197

JUNE 1992 


\section{NOTICE}

This report was prepared as an account of work sponsored by an agency of the United States Government. Neither the United States nor any agency thereof, nor any of their employees, makes any warranty, expressed or implied, or assumes any legal liability or responsibility for any third party's use or the results of such use of any information, apparatus, product or process disclosed in this report, or represents that its use by such third party would not infringe privately owned rights.

Printed in the United States of America Available from

National Technical Information Service

U.S. Department of Commerce

5285 Port Royal Road

Springfield, VA 22161

NTIS Price codes

Printed copy: $\quad$ A02

Microfiche copy: A01 


\title{
LOCKED MODES AND MAGNETIC FIELD ERRORS IN MST
}

\author{
A. F. Almagri, S. Assadi, S. C. Prager, J. S. Sarff, D. W. Kerst \\ Department of Physics \\ University of Wisconsin \\ Madison, Wisconsin 53706
}

In the MST reversed field pinch [Fusion Technology 19,131(1991)] magnetic oscillations become stationary (locked) in the lab frame as a result of a process involving interactions between the modes, sawteeth, and field errors. Several helical modes become phase locked to each other to form a rotating localized disturbance, the disturbance locks to an impulsive field error generated at a sawtooth crash, the error fields grow monotonically after locking (perhaps due to an unstable interaction between the modes and field error), and over the tens of milliseconds of growth confinement degrades and the discharge eventually terminates. Field error control has been partially successful in eliminating locking.

\section{Introduction}

Long wavelength, global magnetic oscillations greatly influence macroscopic behavior of reversed field pinch (RFP) and tokamak plasmas. Such oscillations, usually identified as MHD tearing modes, can cause sawtooth oscillations and major disruptions. These modes are sensitive to the structure of the equilibrium magnetic field. Ordinarily they rotate with respect to the lab frame. However, in the presence of a nonaxisymmetric error in the equilibrium magnetic field the modes can cease rotation and become stationary or "locked" in the lab frame. In recent years, many studies of locked modes in tokamaks have illustrated their deleterious effects on plasma behavior. $1,2,3,4,5$

In the MST reversed field pinch locked modes occur in response to field errors, with similarities to lockirig in tokamaks. 5,6,7 However, the locking in MST is part of a rich process which includes interactions between helical modes, field eirors and sawteeth. We view this wall locking process as a sequence of the following observed events

(1) Phase-locking between modes : The dominant modes in MST (with poloidal and toroidal mode numbers $m=1, n=5-10$ ) become locked in phase to form a rotating, localized, magnetic disturbance with a toroidal width of about one-fourth of the circumference. This is similar to earlier observations of phase-locking in the OHTE 8 and TPE1-RM15 ${ }^{9}$ devices, as well as in MHD computation. 10,11 
(2) Impulsive field error generated by sawtooth crash : The sawtooth crash flattens the current density profile and shifts the plasma inward, generating a sudden radial field error at the poloidial gap in the shell.

(3) Rotating modes lock to field error : Coincident with the sawtooth crash (and impulsive field error), the rotating, phase-locked structure halts at the location of the field error ( poloidal gap ).

(4) Field errors grow after locking : After locking, stationary helical modes are formed and magnetic field error at both the poloidal and toroidal gaps begin to grow. The growth, roughly linear in time, is interpreted as arising from an unstable interaction between the helical modes and the field error at the toroidal gap.

(5) Confinement degrades and discharge terminates prematurely: As the field errors grow for tens of milliseconds, confinement degrades, eventually terminating the discharge.

The modes do not lock at every sawtooth crash, but when they do, this sequence is extremely reproducible and obviously harmful to plasma confinement. Each step in the process is described below. After describing the apparatus in section II, we discuss the observations of mode-mode locking (section III), mode-locking to the wall (section IV), field error growth after locking (section V), and partial control of the locking by field error control (section VI). We summarize in section VII.

\section{Description of Apparatus}

\section{IIA MST Device and Plasma Parameters}

MST is a large toroidal reversed field pinch which, from a field error point of view, contains three unique design features. ${ }^{12}$ First, the toroidal magnetic field is produced by driving poloidal current through the conducting vacuum vessel $(5 \mathrm{~cm}$ thick aluminum, minor radius $=0.52 \mathrm{~m}$, major radius $=1.5 \mathrm{~m})$. This single-turn coil design produces a very low field ripple in vacuum. The dominant spatial Fourier component (poloidal mode number $\mathrm{m}=0$, toroidal mode number $n=4$ ) has an amplitude of $0.5 \%$ of the toroidal field. Second, the poloidal magnetic field windings do not encircle the torus axisymmetrically, but are wrapped about the iron core. Third, vacuum pumping is provided through 193,1-1/2 inch diameter holes in the lower half of the chamber which are spread over nearly the entire toroidal 
circumference. The resulting field error is a small ripple of small spatial scale, as opposed to an error of large radial extent which would accompany a large porthole. The conducting shell contains two insulated voltage gaps. One cut, the toroidal gap, extends the long way around to allow application of the toroidal magnetic field. The poloidal gap extends the short way around the shell to allow the pulsed poloidal magnetic field to enter the shell.

MST discharges, formed in self-reversal mode, have been produced for durations up to $80 \mathrm{~ms}$ and plasma currents up to $0.6 \mathrm{MA}$. MST can operate over the range of plasma parameters depicted in Table 1.

\section{IIB Sources of Field Errors}

The dominant source of field error in MST is the poloidal gap in the vacuum shell. This gap interrupts the flow of toroidal current in the shell surface. This current is an image of the plasma current and must be accurately controlled at the gap. A small poloidal component of the current at the gap can produce a significant radial magnetic field which enters the shell through the $1 \mathrm{~cm}$ wide gap. Such errors generally have low $\mathrm{m}$ values and a very broad range of $n$ values since the gap is localized toroidally. Hence, these errors are usually resonant with the equilibrium field in the plasma. The error at the toroidal gap is enhanced in the presence of plasma; however, the errors are generally not resonant in the plasma and are less deleterious than the other gap.

The errors generated at the poloidal gap can be divided into four categories according to their time dependence. The first type has the same time waveform as the plasma current and results from the poloidal field winding being imperfectly placed for the specific plasma equilibrium. The dominant poloidal mode numbers of this error are $m=0-2$. The second field error begins a slow growth later in time (at about $10-20 \mathrm{~ms}$ ) and is caused by the finite electrical resistivity of the shell. It is dominantly an $\mathrm{m}=4$ error related to the rectangular symmetry of the poloidal field winding. The third type is an oscillatory $m=1$ error generated by the rotating helical modes. The fourth type is an impulsive $m=1$ error generated by the sudden equilibrium inward shift that accompanies the crash phase of a sawtooth oscillation.

The poloidal gap is instrumented with two sets of correction coils (saddle coils) to reduce the first two types of field errors. Current in the first set of correction coils is driven in series with the poloidal field winding to produce a correction proportional to the plasma current. Radial field of the first type penetrating the gap is thereby reduced to $1 \%$ of the equilibrium field. The second set is driven by a simple waveshaping circuit to match the slowly varying error of the second type. The third and the fourth type of error occurs on a sub-millisecond timescale and, at present, are not corrected. Unfortunately, the fourth type of field error plays an important role in modelocking to the wall. 
IIC Magnetic Diagnostics

About 400 magnetic pickup coils are attached to the inside surface of the vacuum shell to diagnose the magnetic fluctuations and gap field errors. The helical modes have also been diagnosed by other methods, such as soft xray emission, 13 which will not be described here. To measure the field errors, 32 coils are distributed around the poloidal gap, and 16 coils are distributed around the toroidal gap. These coils detect the radial magnetic field at the gaps, with mode numbers up to $\mathrm{m}=16$ at the poloidal gap and mode numbers up to $\mathrm{n}=8$ at the toroidal gap. To measure the toroidal mode spectrum, coils are located at 64 toroidal locations, equally spaced, with three orthogonal coils at each location (for each directional component). Another set of 16, 3-axis coils is distributed poloidally to measure the poloidal mode spectrum. Thus, magnetic fluctuations can be detected with mode numbers up to $m=8$ and $n=32$. The signals are typically amplified with a $250 \mathrm{kHz}$ bandwidth and digitized up to a $1 \mathrm{MHz}$ sampling rate.

III Mode-Mode Phase Locking

Magnetic fluctuations in MST are dominated by low frequency components $(10 \mathrm{kHz}-30 \mathrm{kHz})$ which contain about $90 \%$ of the power. $14,15,16$ These fluctuations, identified as tearing modes, are composed of several spatial modes with $m=1, n=5-10$. Typically these modes phase-lock to each other and constructively interfere to produce a rotating, toroidally localized disturbance. The localization and rotation is clear in Fig. 1a, which displays the time dependence of $\widetilde{B}$ at 32 different toroidal locations. Usually the disturbance extends over about one-fourth of the toroidal circumference and rotates in the ion diamagnetic direction at about $10^{6} \mathrm{~cm} / \mathrm{sec}$. However, the speed, and even the direction, can vary with time depending on the modal content of the spectrum. The phase-locking between modes is a result of nonlinear coupling, as has been identified through bi-spectral analysis of magnetic fluctuations in MST. 17

The rotating disturbance produces a field error at both gaps (of the third type described in section IIB). The poloidal field windings are distributed for axisymmertic shell image currents corresponding to a particular plasma equilibrium. The tearing modes produce helical image currents in the shell. The mismatch between the winding distribution and image currents yields a radial field at the poloidal gap as the rotating disturbance passes the gap. This is illustrated in Fig. 1b. The field error at the gap is an $m=1$ component, as experted since it is generated by the $m=1$ modes. Similarly, the rotating disturbance induces a localized error field at the toroidal gap, shown in Fig. 2.

MST and other RFP experiments displays vivid sawtooth oscillations. 18 The data described above correspond to times during the rise phase of a sawtooth. At the crash, the magnetic fluctuation spectrum broadens 
drastically ( $n>32)$, and the spectrum cannot be accurately resolved. After the crash, the toroidal spectrum is composed of only $n=5-7$ with $n=5$ being typically the dominant mode. The localized structure slowly reforms as the other low frequency modes $(n=6-10)$ begin to grow and the $n=5$ mode decays. Eventually the dominant modes phase lock to produce a highly localized structure that remains localized until the following sawtooth event. This cycle, displayed in Fig. 3, repeats throughout the discharge. Occasionally the $\mathrm{n}=5-7$ modes become locked to the wall at the crash and remain stationary for the remainder of the discharge with same modal content.

\section{Mode-Locking to the Wall}

A sawtooth crash generates an impulsive field error at the poloidal gap (of the fourth type described in section IIB). The current density profile flattens during a sawtooth crash. This is indicated in measurements of the etge poloidal current density (which is roughly parallel to the magnetic field) in the edge plasma, shown in figure 4, with a small Rogowskii coil. At a crash the current density doubles, while the total plasma current remains nearly unchanged. The current flattening shifts the plasma inward, as indicated by the decrease in the edge poloidal magnetic field asymmetry factor given by $B_{\theta}=B_{0}(1+\varepsilon \Lambda \cos \theta)$, as shown in figure $5 \mathrm{a}$. As a result of the sudden change in the equilibrium, the poloidal field windings become mis-matched, and an impulsive $m=1$ radial field error is produced, as shown in figure $5 b$.

At the time of a sawtooth crash, the helical modes sometimes cease rotation and the localized structure remains at the location of the poloidal gap for the remainder of the discharge, as shown in figure 6 . The locking of the disturbance is observed in the toroidal coil array. The toroidal angle at which the mode locks corresponds to the poloidal gap, and the time of locking corresponds to the sawtooth crash. This locking of the mode to the wall results from the interaction between the mode and the impulsive magnetic field error at the poloidal gap. The detailed mechanism for locking (e.g. viscous damping, impurity generation from enhanced plasma-wall interaction or magnetic boundary conditions ) has not been determined. The locking substantially alters the discharge evolution and leads to early termination as seen in figure 7.

\section{Field Error Growth After Locking to the Wall}

After the helical modes lock to the wall, they remain locked and large until discharge termination. This is in contrast to the usual case where the modes do not lock and reset to zero at each crash. In figure 8 we show the time evolution of the dominant Fourier modes, $n=5-7$. The modes become stationary at $11 \mathrm{~ms}$. The stationary modes are also evident as a growing field error. At the poloidal gap a growing $m=1$ error is observed, and at the toroidal gap the growing error consists of $n=5-7$ modes as seen in figure 9 . 
The growth of the field errors at the gaps after the toroidal modes become stationary might arise from an instability generated from the interaction of the helical mode with the toroidal gap. Such a "field error instability" was predicted in a heuristic model by Kerst. ${ }^{19} \mathrm{~A}$ helical plasma current ( such as produced by a tearing mode) will induce a radial magnetic field at the toroidal gap with the same toroidal mode number as the tearing mode. As the radial magnetic field enters the plasma it diverges spatially, producing a toroidal magnetic field perturbation, $\delta \mathrm{B}_{\mathrm{t}}$. The resulting Lorentz force, $\mathrm{J}_{\mathrm{P}} \mathrm{X} \delta \mathrm{B}_{\mathrm{t}}$, produces a radial force pushing the initial helical distortion toward the toroidal gap, unstably enhancing the original perturbation.

Consider a plasma current column in a large aspect ratio toroidal shell with a minor radius, $r_{0}$, major radius, $R$, and wall thickness, $d$, all in units of $\mathrm{cm}$. Let the shell have one toroidal gap located at the inner midplane of width, $\mathrm{G}$, in $\mathrm{cm}$. If the current is displaced vertically with toroidal modulation of $y=y_{0} \sin (n \varnothing)$ where $y_{0}$ is the vertical displacement, in $\mathrm{cm}$, then the radial field, $\delta B_{r}$, in gauss, generated at the toroidal gap is given by

Where I is the plasma current.

$$
\delta B_{r}(\varnothing)=0.4 \pi\left(\frac{y_{0 I}}{2 \pi r_{0} d}\right) \sin (n \varnothing) .
$$

If we assume that this flux fills the volume of the torus, then a toroidal field perturbation, $\delta \mathrm{B}_{\mathrm{t}}$, will be produced

$$
\delta B_{t}(\varnothing)=0.2\left(\frac{y_{0} I R G}{\pi d n r_{0}^{3}}\right) \cos (n \varnothing) .
$$

The total radial force on the current I toward the toroidal gap due to its transverse undulation across $\delta \mathrm{B}_{\mathrm{t}}$ is then given by

$$
\mathrm{F}=\frac{\mathrm{I}^{2} \mathrm{G} \mathrm{y}_{0}^{2} \mathrm{R}}{10 \mathrm{r}_{0}^{3} \mathrm{nd}}
$$

and the restoring force resulting from the conducting wall is given by

$$
\mathrm{F}_{\mathrm{r}}=\frac{\pi \mathrm{R}}{\mathrm{n}}\left(\frac{0.2 \mathrm{I}^{2} \mathrm{y}_{0}}{\mathrm{r}^{2}}\right) \text {. }
$$

The ratio of the perturbation force, $F$, to the restoring force, $F_{r}$, is

$$
\frac{F}{F_{r}}=\frac{G y_{0}}{2 \pi r_{0} d}<\frac{G}{2 \pi d}=4 \% \text { (For MST ) }
$$

These forces are present throughout the discharge. However, if the modes are stationary, the image currents in the shell resistively decay, and the restoring force is reduced. At the same time, the column moves closer to the gap, and the effective gap width, G, increases, and the effective wall thickness, $d$, reduces, thereby enhancing the driving force.

The equivalent vertical magnetic field, $B_{v}$, that would produce the same radial force toward the toroidal gap is given by

$$
\mathrm{B}_{\mathrm{v}} \leq \frac{\mathrm{B}_{\mathrm{p}}}{2 \pi}\left(\frac{\mathrm{G}}{\mathrm{d}}\right)
$$

This vertical field is observed as an increasing error field at the poloidal gap as the factor $\mathrm{G} / \mathrm{d}$ increases. 


\section{Control of Locking to the Wall}

Since the locking is believed to be caused by the impulsive field error at the poloidal gap during the sawtooth crash, control of locking is possible through field error control. This is quite difficult since such control would require feedback of the magnetic field at the gap on the sub-millisecond timescale of the crash. However, we have been able to eliminate the locking in some discharges by imposing an steady $m=1$ radial field at the gap in the direction opposite to the impulsive error. Although the steady field increases the field error at times other than during the crash, it diminishes the error during the crash. As a result the impulsive error is reduced to a level at which locking does not occur, as illustrated in figure 10. In MST locking is most prevalent at high plasma current $(400 \mathrm{kA}$ to $600 \mathrm{kA})$. The effectiveness of the offset error diminishes with current. At the highest currents locking is often unavoidable. Presumably if the sawtooth oscillations are eliminated, then locking would not occur. It is anticipated that improved flat-toping of the discharge might eliminate inductive contributions to the current density profile to form a more sawtooth-stable discharge.

VII Summary

A process has been observed in MST which culminates with the monotonic growth of a locked mode which eventually terminates the discharge. The locking of tearing modes in MST has obvious similarities to locked modes in tokamaks. In both cases, locked modes can occur at any time during the discharge (current rise or flat-top), can be caused by field errors, can be initiated by a sawtooth crash, and lead to degraded confinement. One difference is that locked modes in tokamaks can unlock during a discharge, whereas in MST the modes remain locked until termination.

However, the helical modes, sawteeth, and field errors couple in MST in ways not reported in tokamaks. Firstly, in tokamaks locked modes usually are composed of only one helical mode. In RFPs several tearing modes are present simultaneously and become phase-locked, as a result of nonlinear mode coupling. In most tokamaks the field error is a dc pre-existing error, whereas in MST it is generated by the sawtooth crash (which is itself likely caused by the phase-locked tearing modes). The sawtooth-generated impulsive field error then causes the rotating modes to lock to the field error. Unlike tokamak locked modes, after locking in MST the error fields grow monotonically, perhaps for $10 \mathrm{~ms}$ to $30 \mathrm{~ms}$, eventually terminating the discharge. We conjecture that the growth after locking is generated by an unstable interaction between the field error and the helical mode (a "field error instability").

Locked modes are a detriment to confinement. Two possible means to eliminate locking are to suppress the field errors or to eliminate sawteeth. 
We have applied steady field opposite to the impulsive error. As a result of this offsetting field, the impulsive errors are diminished and locking is sometimes eliminated. However, it is more desirable to eliminate the sawtooth oscillations, the source of the impulsive field error. MST displays strong sawteeth in nearly all plasmas, in contrast to other RFPs which are sawtooth-dominated only at high values of pinch parameter. A possible reason is that the current in MST is not sufficiently time-independent during the discharge, and inductive effects produce a current density profile more prone to sawteeth. The MST power supplies are being modified for an improved flat-top to test this speculation. On the positive side, the sensitivity of tearing modes to the magnetic field boundary conditions suggests the possibility of controlling the modes through edge control.

Acknowledgments

This work is supported by the U.S. Department of Energy. 


\section{References.}

1 J. T. Scoville, R. J. LaHaye, A. G. Kellman, T. H. Osboren, R. D. Stambaugh, E. J. Strait, T. S. Taylor, Nuclear Fusion, Vol. 31, No. 5 (1991).

2 M. F. F. Nave, J. A. Wesson, Nuclear Fusion, Vol. 30, No. 12 ( 1990 )

3 H. Ninomiya, K. Itami, Y. Neyatani, O. Naito, R. Yoshino Nuclear Fusion, Vol. 28, No. 7(1988).

4 Snipes J. A., Campbell D. J., et al.

Nuclear Fusion, Vol. 28, No. 6(1988).

$5 \quad$ Karger F., Wobig H., Corti S., et al.

Plasma Physics and controlled Nuclear Fusion Research 1974

(Proc. 5th Int. Conf. Tokyo,1974), Vol. 1, IAEA, Vienna(1975)207.

6 Bol K., Cecchi J. L., Daughney C. C., et al.

Plasma Physics and controlled Nuclear Fusion Research 1974

(Proc. 5th Int. Conf. Tokyo,1974), Vol. 1, IAEA, Vienna(1975)83.

7 Johnson P. C., Allen J., Axon K.B., et al.

Plasma Physics and controlled Nuclear Fusion Research 1984

(Proc. 10th Int. Conf. London,1984), Vol. 1, IAEA, Vienna(1985)239.

$8 \quad$ R. LaHaye et. al., Phys. Fluid, 27,2576(1984).

9 K. Hattori, Y. Hirano, T. Shimada, Y. Yagi, Y. Maejima, I. Hirota, and K. Ogawaet, Phys. Fluids B 3 (11), November 1991.

10 D. D. Schnack and S. Ortolani, Nuclear Fusion, 30,227(1990).

11 Kusano, et. al., 13th Int. Conf. on plasma Physics and controlled Nuclear Fusion Research(1990). 
12 R. N. Dexter, D. W. Kerst, T. W. Lovell, S. C. Prager, and J. C. Sprott, Fusion Technology 19,131(1991).

13 G. Charts, Ph.D. Thesis, University of Wisconsin-Madison (1991). and G. Charts, S. A. Hokin, submitted for publication

14 S. C. Prager, A. F. Almagri, S. Assadi, J. A. Beckstead, G. Charts, R. N. Dexter, D. J. Den Hartog, S. A. Hokin, T. W. Lovell, T. D. Remple, J. S. Sarff, W. Shen, C. W. Spragins, and J. C. Sprott, Phys. Fluids B 2,1367(1990).

15 A. F. Almagri, S. Assadi, J. A. Beckstead, G. Charts, N. Crocker, R. N. Dexter, D. J. Den Hartog, S. A. Hokin, D. Holly, E. Nilles, S. C. Prager, T. D. Remple, J. S. Sarff, E. Scime, C. W. Spragins, J. C. Sprott, G. Starr, M. Stoneking, and C. Watts, Edge Fluctuation in the MST Reversed Field Pinch Workshop of Physics of Alternative Magnetic Confinement Schemes, Verenna, Italy, 1990.

16 J. S. Sarff, A. F. Almagri, S. Assadi, J. A. Beckstead, G. Charts, N. Crocker, D. J. Den Hartog, S. A. Zlokin, D. Holly, E. Nilles, S. C. Prager, T. D. Remple, E. Scime, W. Shen, C. W. Spragins, J. C. Sprott, G. Starr, M. Stoneking, and C. Watts, Edge Fluctuation and Transport in the MST Reversed Field Pinch. Controlled Fusion and Plasma Physics (Proc. 18th Eur. Conf. Berlin,1991), Vol. 15 C, Pt II, European Physical Society(1991)289.

17 S. Assadi, Ph.D. Thesis University of Wisconsin-Madison (1992). and S. Assadi, S. C. Prager, K. L. Sidikman, submitted to Phys. Rev. Lett.

18 J. A. Beckstead, Ph.D. Thesis University of Wisconsin-Madison (1990).

19 D. W. Kerst, Uriversity of Wisconsin-Madison, Report PLP\#909(1983). 
TABLE 1. Limiting values of plasma parameters achieved on MST

$\begin{array}{lll}\mathrm{Ip} & < & 0.6 \mathrm{MA} \\ \mathrm{Vl} & > & 12 \mathrm{~V} \\ \tau_{\mathrm{pulse}} & < & 80 \mathrm{~ms} \\ \mathrm{Te}(0) & & 50-430 \mathrm{ev} \\ \operatorname{ne}(0) & & 0.75-3 \times 10^{13} \mathrm{~cm}^{-3} \\ \mathrm{Ti} & < & 350 \mathrm{ev} \\ \beta \mathrm{p} & & 4-17 \%\end{array}$




\section{Figure Captions}

Figure 1. (a) The poloidal magnetic field fluctuation at 32 toroidal locations plotted in their toroidal order as function of time. This plot shows the toroidal extent, toroidal speed, and direction of the localized structure. This is a case in which the toroidal structure is highly localized with a broad modal content $(n=6-10)$. Around $19 \mathrm{~ms}$, the toroidal structure is less localized as fewer number of modes are present. (b)The amplitude of the $m=1$ component of the magnetic field error at the poloidal gap for the same time window as in part $a$. The $m=1$ amplitude is enhanced as the localized structure cross the poloidal gap ( at zero toroidal angle ).

Figure 2. The field error at the toroidal gap at 16 toroidal locations plotted in their toroidal order versus time. This plot show a rntating, localized error correlated with the rotating tearing modes. This error results from the interruption of the poloidal shell current at the gap.

Figure 3. The poloidal magnetic field at 32 toroidal locations versus time, and the field reversal parameter, F, for one sawtooth cycle. The spectrum starts narrow where only few modes are present $(n=5-7)$ resulting in a broad disturbance. Thereafter the spectrum broadens $(n=6-10)$ and forms a localized toroidal structure until the foilowing sawtooth crash. After the crash, the spectrum is narrow and the dominant mode is $n=5$.

Figure 4. The poloidal current density at the edge of the plasma versus time. The sawtooth crash causes the current density to double at the edge, while the total plasma current remain constant.

Figure 5.(a) The poloidal asymmetry factor $\Lambda$ versus time. $\Lambda$ decrease at the sawtooth crash showing the flattening and inward shift the plasma current channel. The change in $\Lambda$ is due mostly to the change in the internal inductance not a change of poloidal beta, which would require beta to triple its value. (b) The $m=1$ component of the magnetic field error at the poloidal gap. Showing an increase in the $m=1$ component correlated with the change in $\Lambda$.

Figure 6. The poloidal magnetic field fluctuation at 32 toroidal locations versus time, showing the locking at the poloidal gap at about $11 \mathrm{~ms}$.

Figure 7. The plasma current for two identical discharges except that one discharge is locked and the other is not. The locked discharge is degraded. 
Figure 8. The amplitudes and phases of the $n=5-7$ tearing modes. The modes lock around $11 \mathrm{~ms}$. The amplitudes are about the same as when the modes are rotating. In all cases the spectrum is narrow after a sawtooth crash $(n=5-7)$. When the modes are locked, only $n=5-7$ are ever large.

Figure 9. The $\mathrm{m}=1$ component of the magnetic field error at the poloidal gap for two identical discharges except that one discharge is locked and the other is not. Once the modes lock, the $m=1$ field error starts to grow to large value eventually terminating the discharge.

Figure 10. The $m=1$ component of the magnetic field error at the poloidal gap with a negative offset. The offset is to prevent the sawtooth crash from driving the impulsive field error, $m=1$, to a large positive value. This was fou id to be successful in preventing locked discharges.( compare with fig. 5b) 


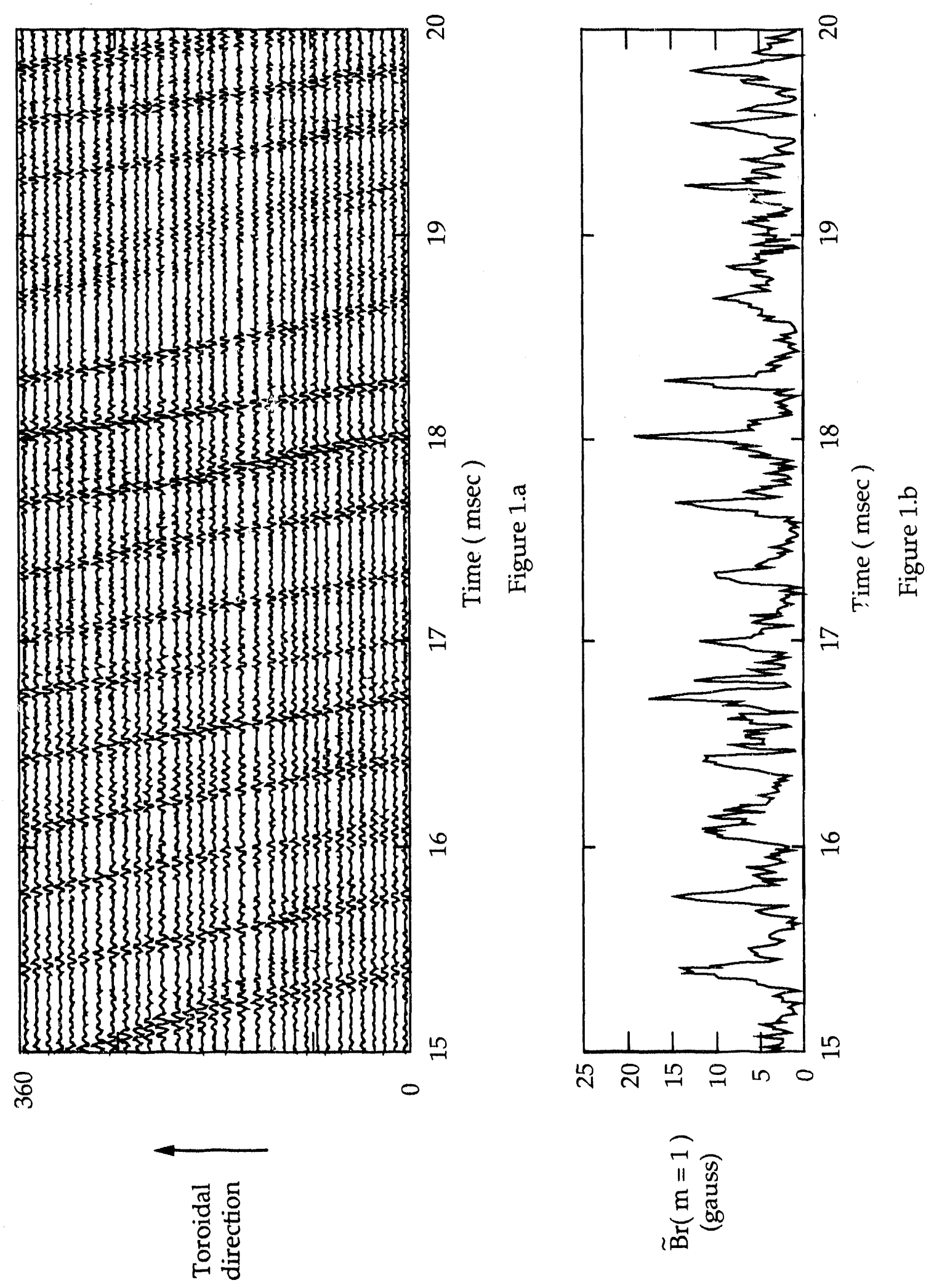




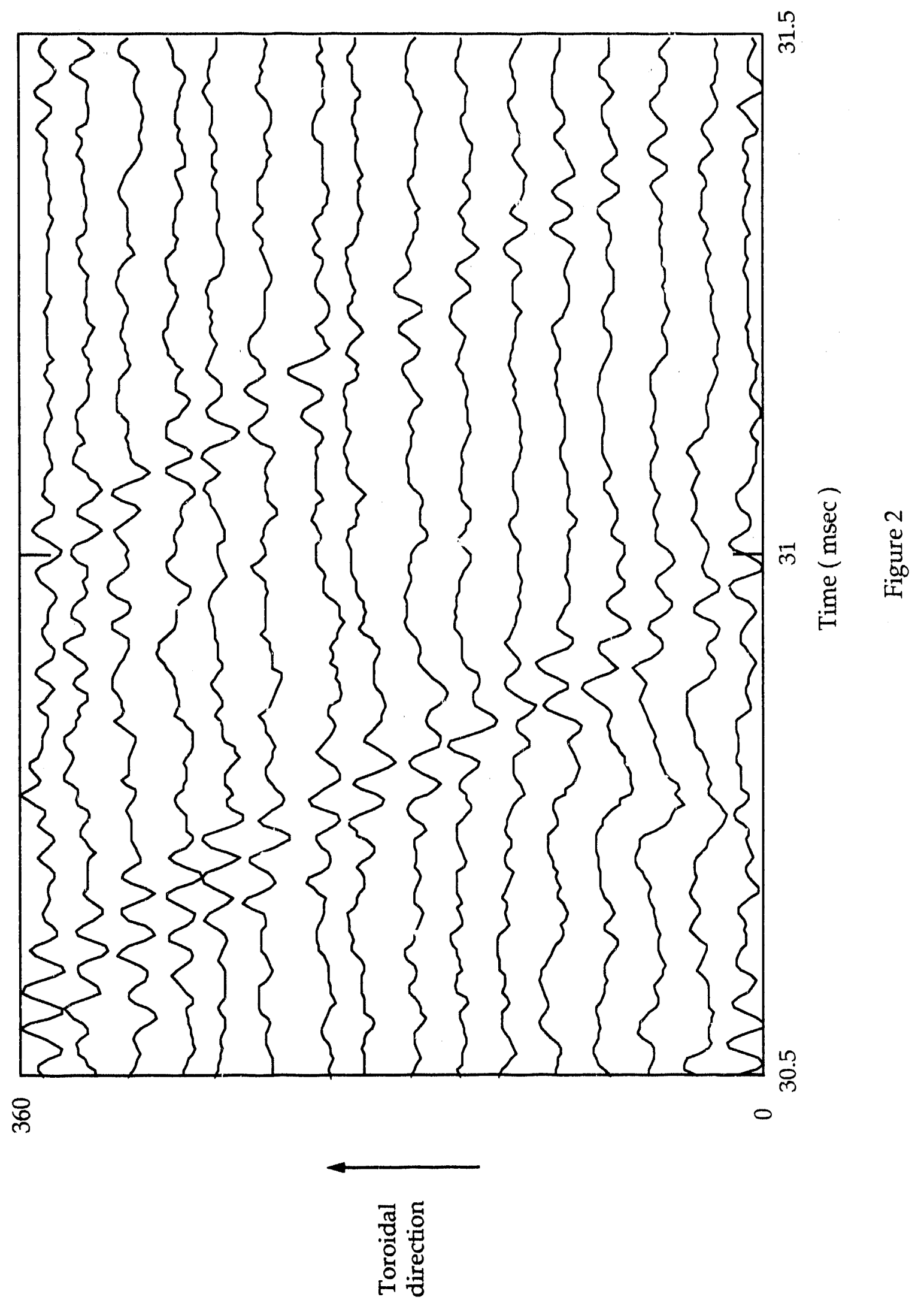




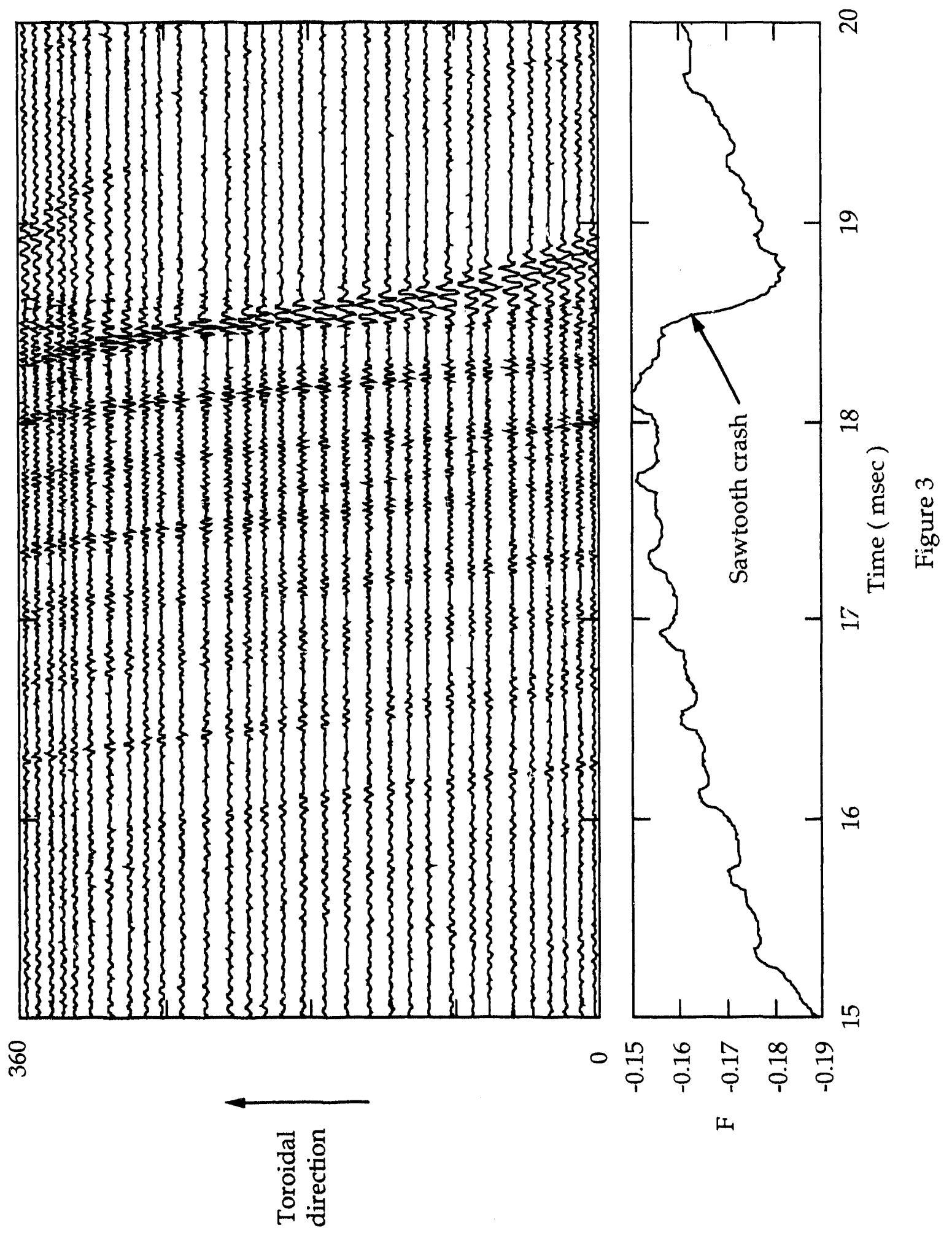




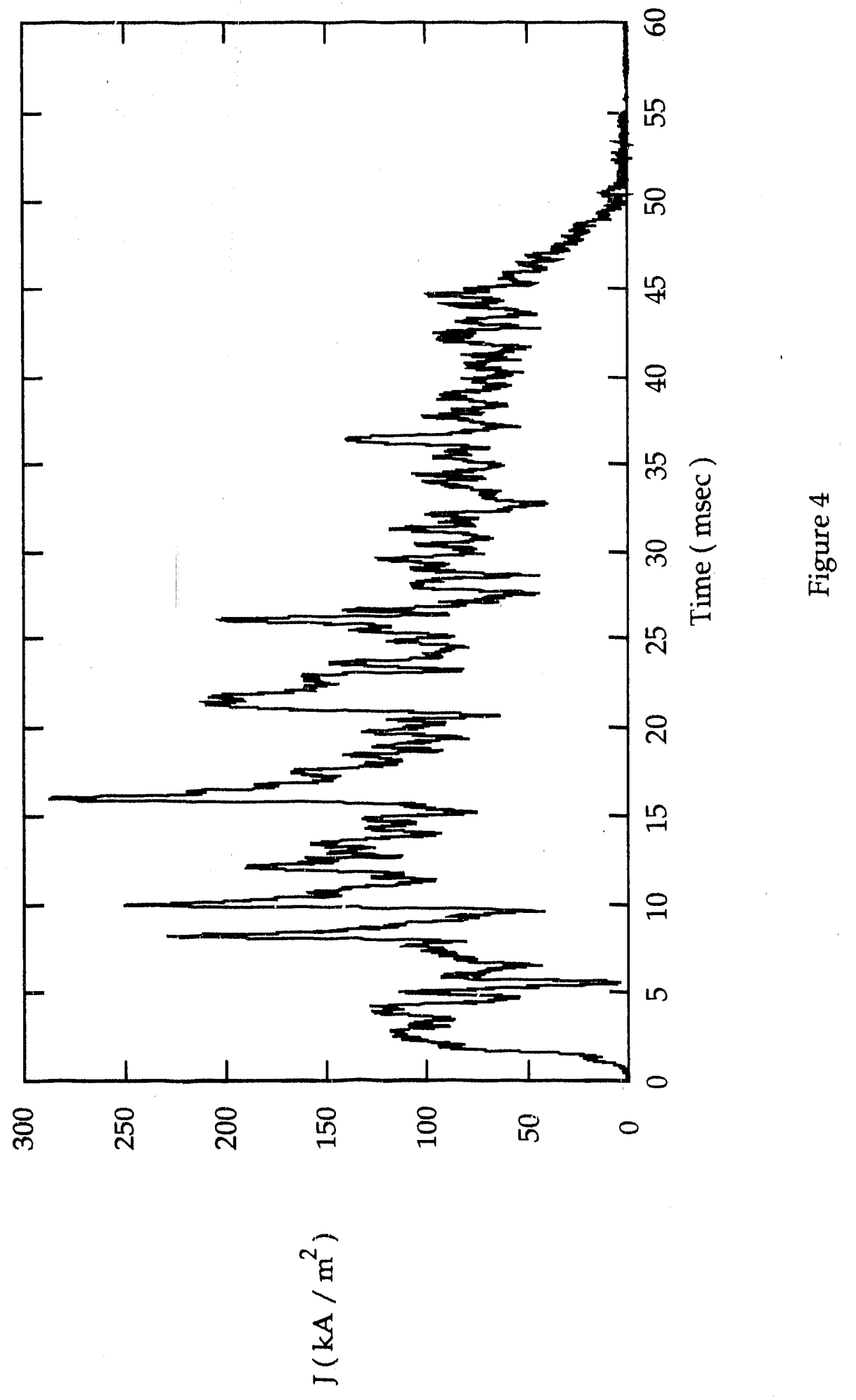



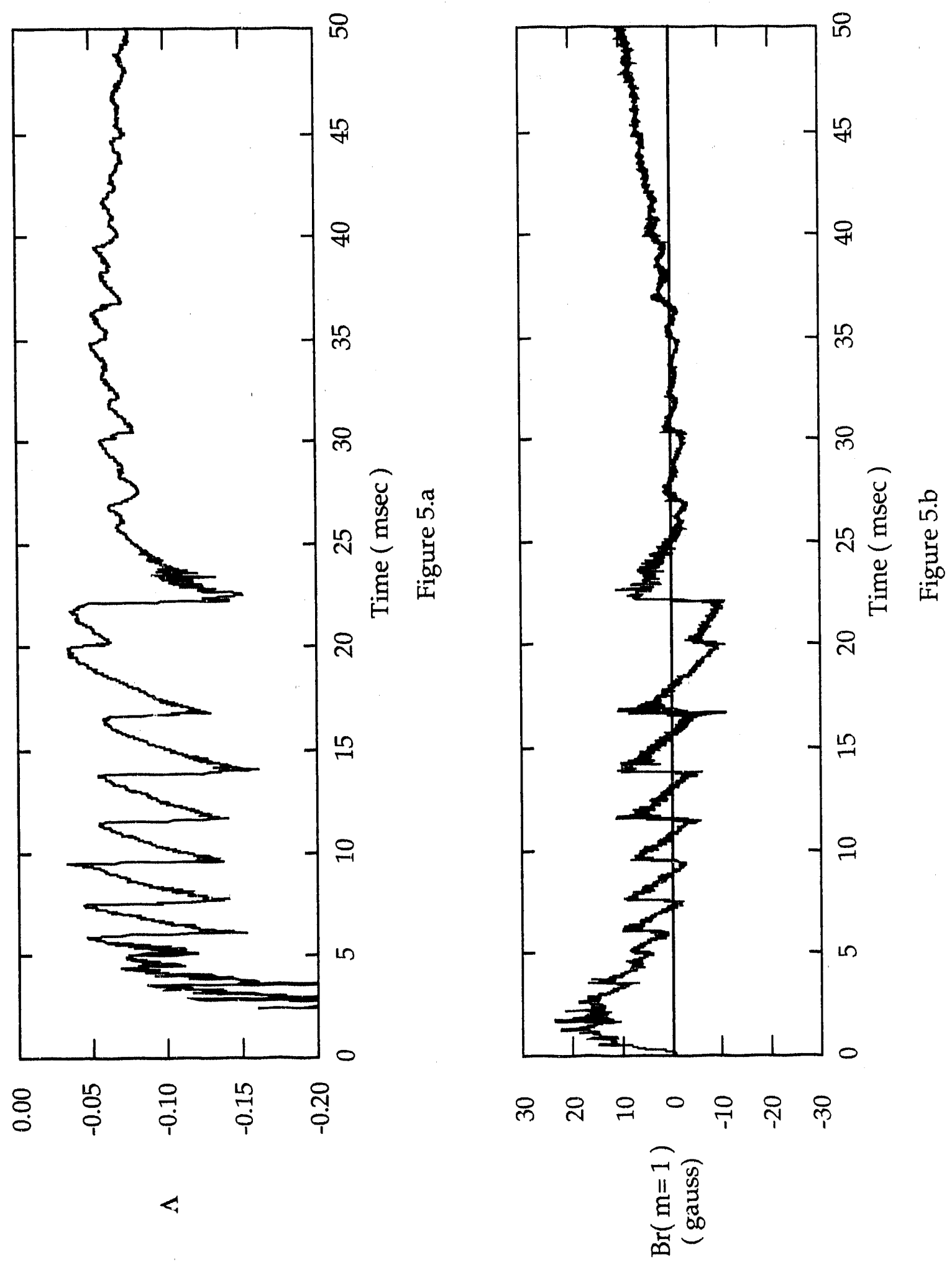


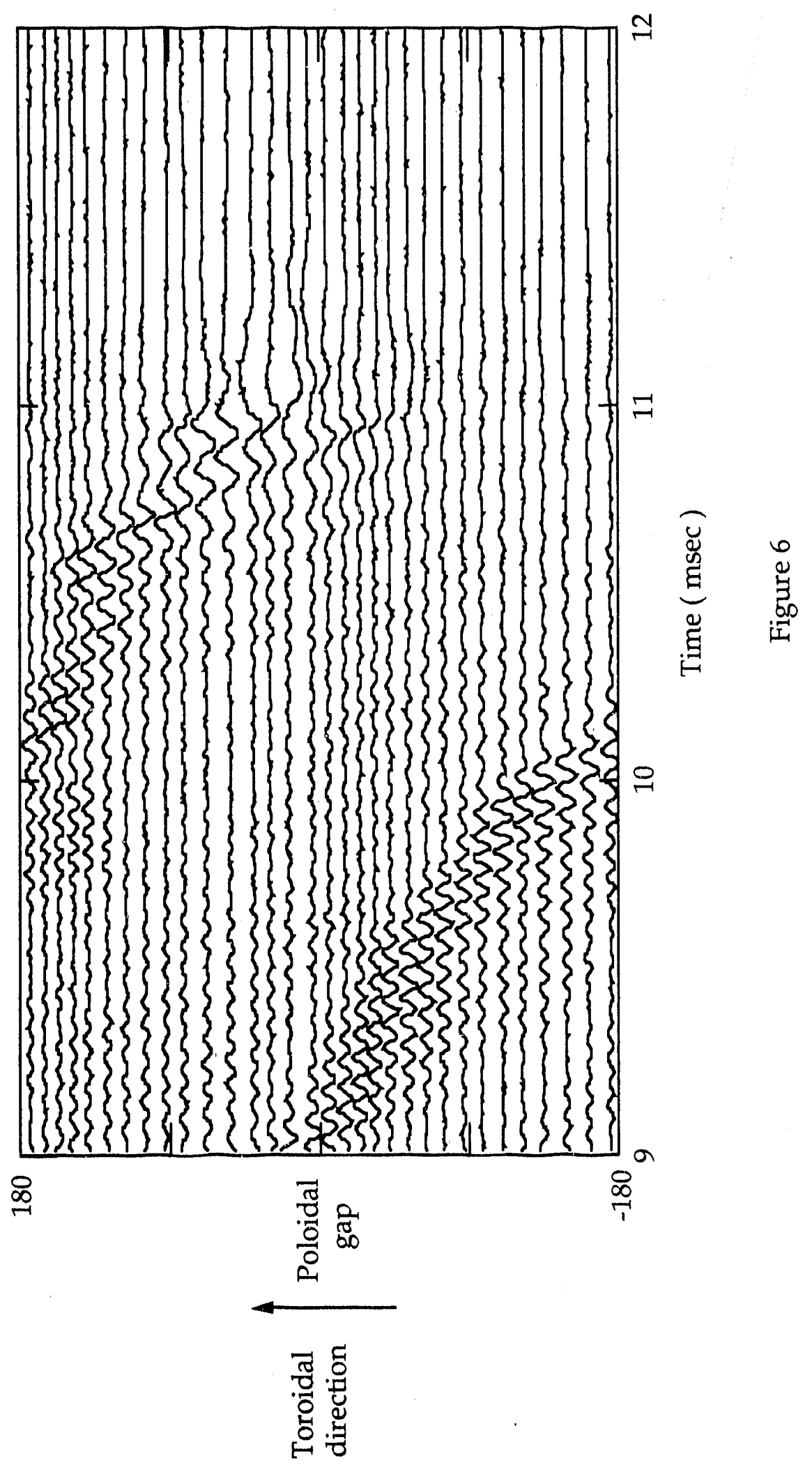




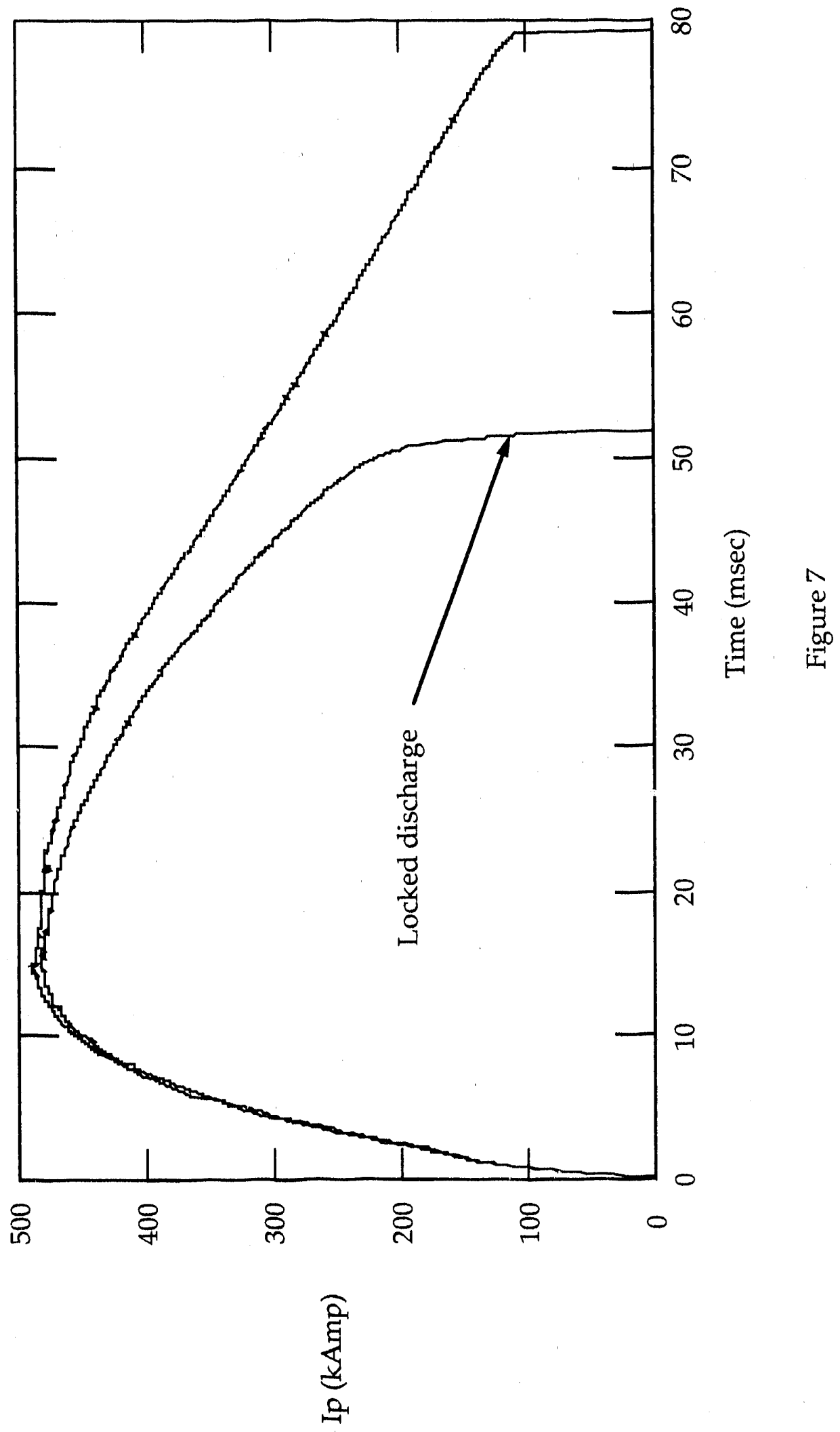




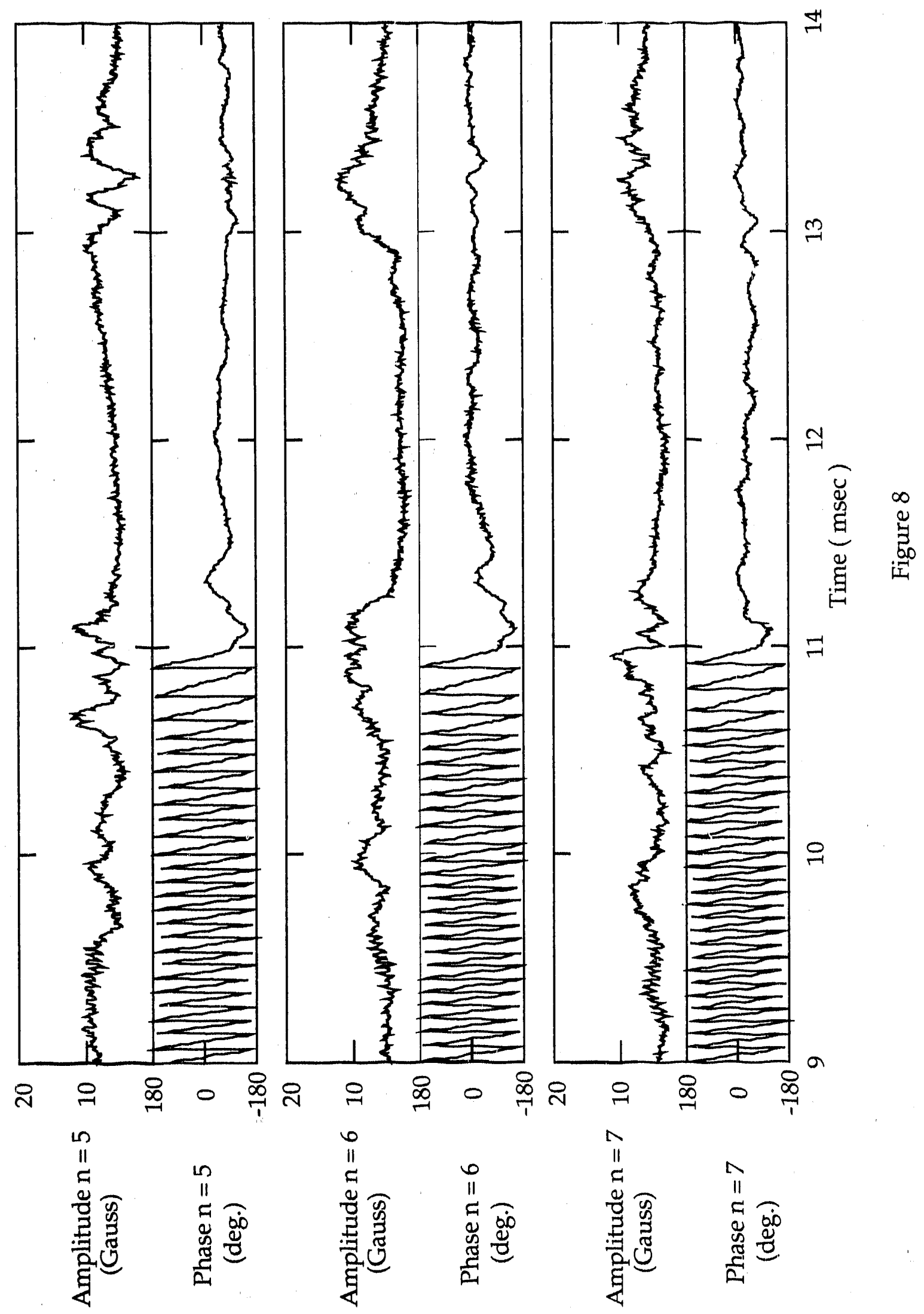




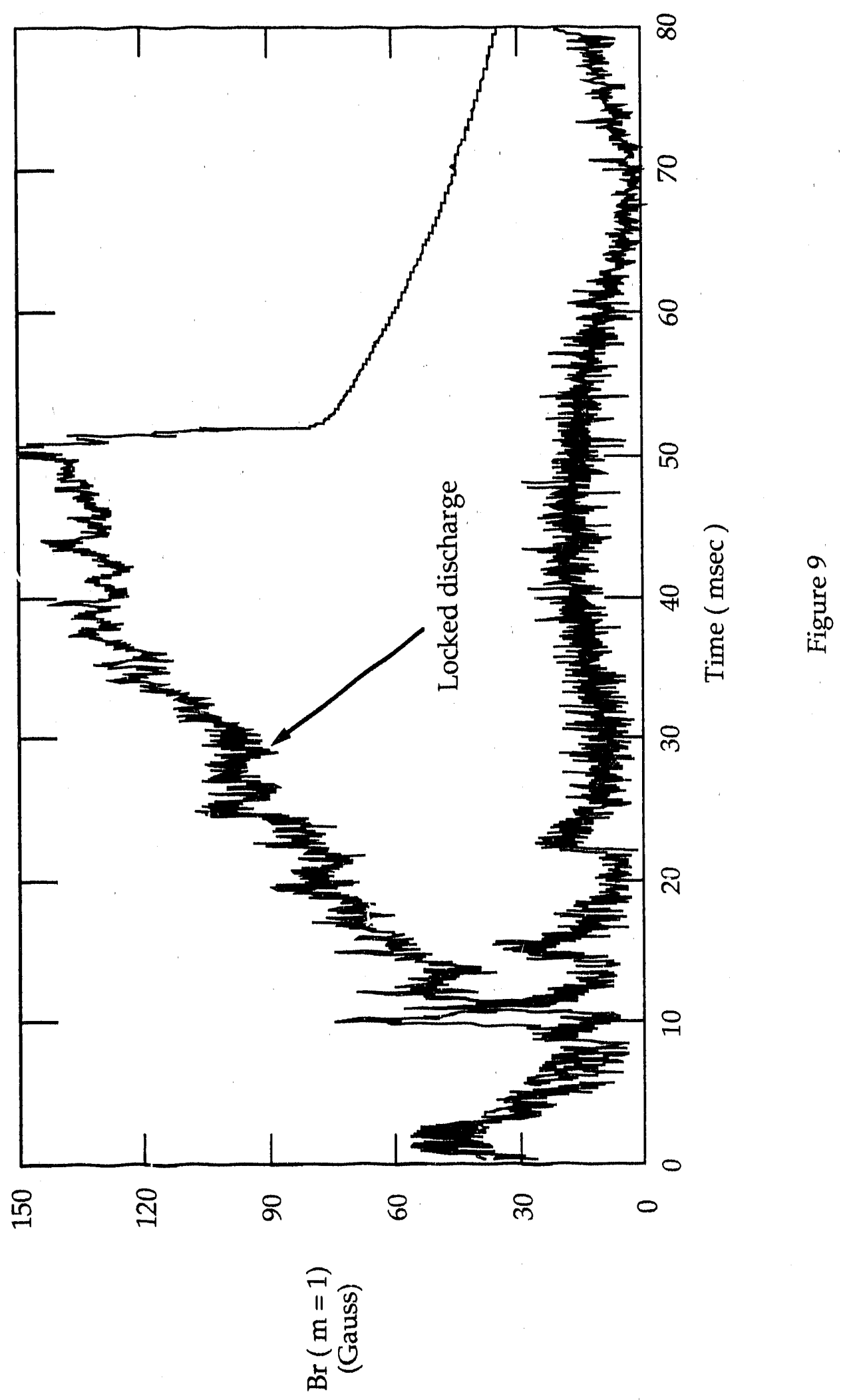




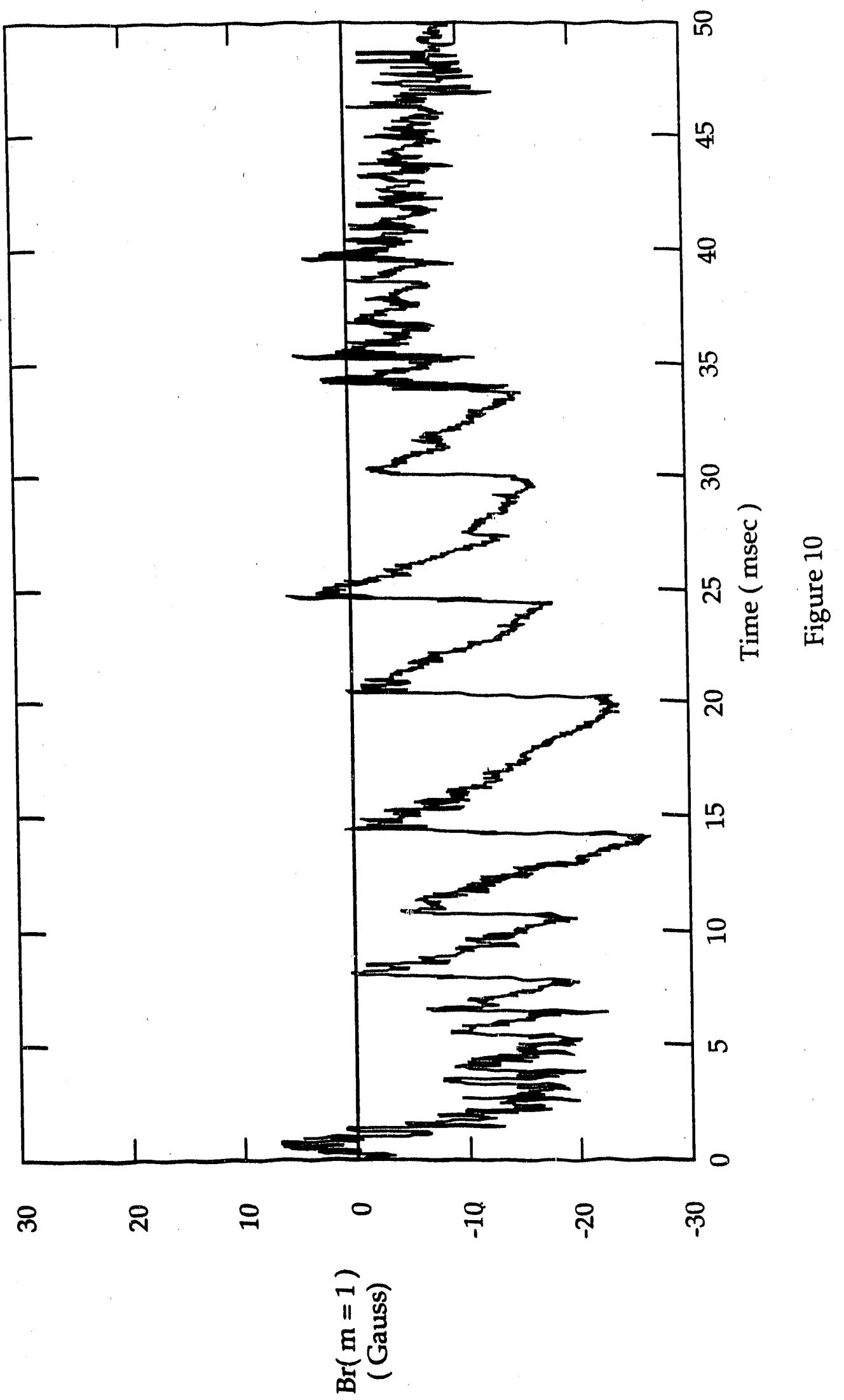




\section{EXTERNAL DISTRIBUTION IN ADDITION TO UC-20}

S.N. Rasband, Brigham Young University

J.B. Taylor, Institute for Fusion Studies, The University of Texas at Austin

M.A. Abdou, University of California, Los Angeles

R.W. Conn, University of California, Los Angeles

T. Dolan, INEL

R. Smith, University of lowa

F.W. Perkins, PPPL

O. Ishihara, Texas Technical University

P.E. Vandenplas, Association Euratom-Etat Belge, Belgium

Centro Brasileiro de Pesquisas Firicas, Brazil

P. Sakanaka, Institute de Fisica-Unicamp, Brazil

Mme. Monique Bex, GANIL, France

J. Radet, CEN/CADARACHE, France

University of loannina, Greece

S. Ortolani, Istituto Gas Ionizzati, EURATON-ENEA-CNR Association, Italy

R. Andreani, Associazione EURATOM-ENEA sulla Fusione, Italy

Y. Kondoh, Gunma University, Kiryu, Gunma, Japan

H. Toyama, University of Tokyo, Japan

FOM-Instituut voor Plasmafysica "Rijnhuizen", The Netherlands

Z. Ning, Academia Sinica, People's Republic of China

P. Yang, Shandong University, People's Republic of China

S. Zhu, University of Science \& Technology of China, People's Republic of China

I.N. Bogatu, Institute of Atomic Physics, Romania

M.J. Alport, University of Natal, Durban, South Africa

R. Storer, The Flinders University of South Australia, South Australia

B. Lehnert, Royal Institute of Technology, Sweden

Librarian, CRPP, Ecole Polytechnique Federale de Lausanne, Switzerland

B. Alper, Culham Laboratory, UK

A. Newton, Culham Laboratory, UK

6 for Chicago Operations Office

4 for individuals in Washington Offices

INTERNAL DISTRIBUTION IN ADDITION TO UC-20

80 for local group and file 

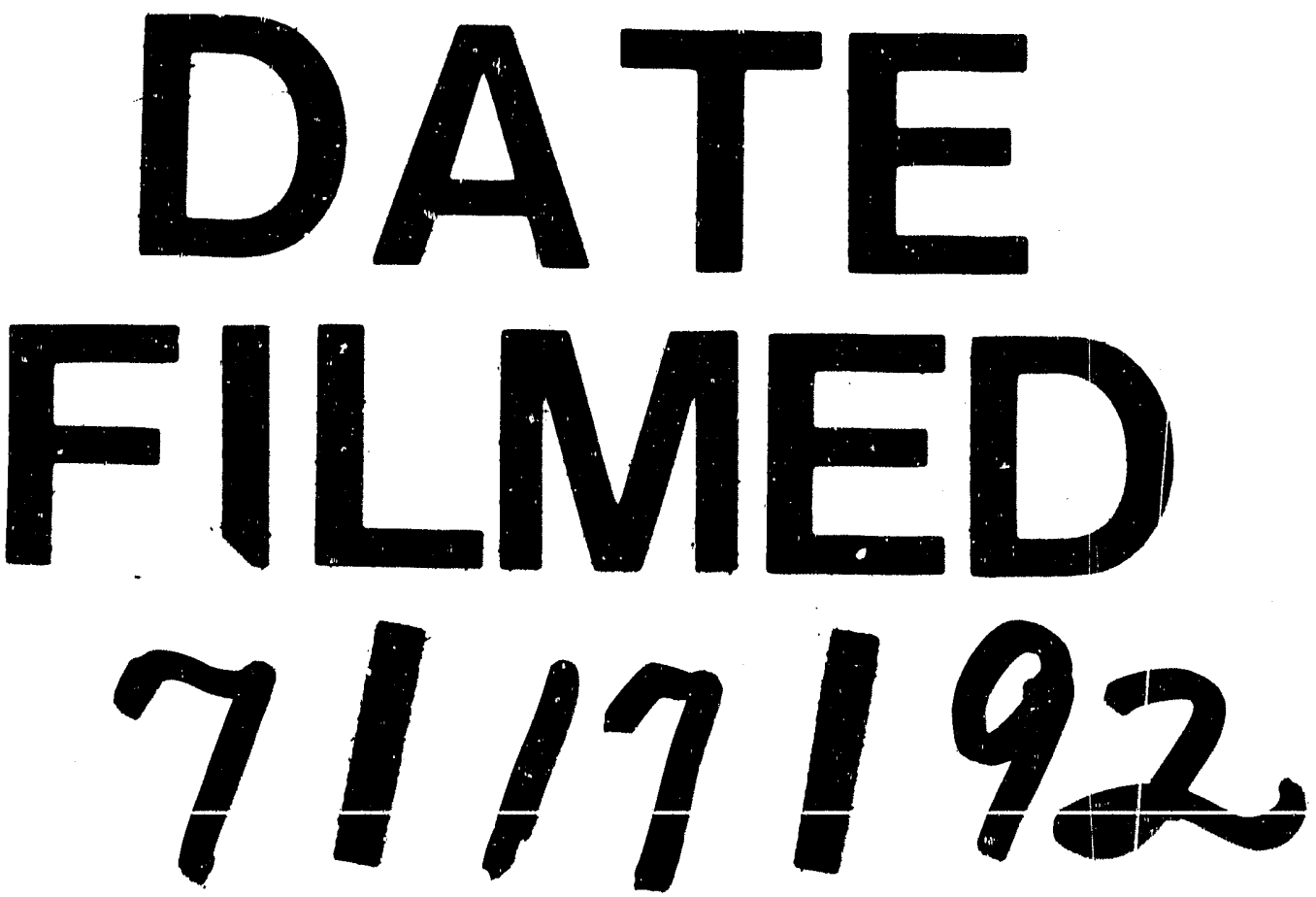

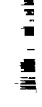


$\underline{\underline{\underline{\underline{\underline{z}}}}}$ 